Solving the Accelerator-Condenser Coupling Problem in a Nanosecond Dynamic Transmission Electron Microscope

B. W. Reed, T. LaGrange, R. M. Shuttlesworth, D. J. Gibson, G. H. Campbell, N. D. Browning

January 11,2010

Review of Scientific Instruments 
This document was prepared as an account of work sponsored by an agency of the United States government. Neither the United States government nor Lawrence Livermore National Security, LLC, nor any of their employees makes any warranty, expressed or implied, or assumes any legal liability or responsibility for the accuracy, completeness, or usefulness of any information, apparatus, product, or process disclosed, or represents that its use would not infringe privately owned rights. Reference herein to any specific commercial product, process, or service by trade name, trademark, manufacturer, or otherwise does not necessarily constitute or imply its endorsement, recommendation, or favoring by the United States government or Lawrence Livermore National Security, LLC. The views and opinions of authors expressed herein do not necessarily state or reflect those of the United States government or Lawrence Livermore National Security, LLC, and shall not be used for advertising or product endorsement purposes. 
LLNL-JRNL-422308

\title{
Solving the Accelerator-Condenser Coupling Problem in a Nanosecond Dynamic Transmission Electron Microscope
}

B. W. Reed, ${ }^{1}$ T. LaGrange, ${ }^{1}$ R. M. Shuttlesworth, ${ }^{1}$ D. J. Gibson, ${ }^{1}$ G. H. Campbell, ${ }^{1}$ and N. D. Browning ${ }^{1,2}$

${ }^{1}$ Lawrence Livermore National Laboratory, Livermore, CA 94551, USA

${ }^{2}$ Department of Chemical Engineering and Materials Science, University of California, Davis, Davis, CA 95616, USA

\begin{abstract}
We describe a modification to a transmission electron microscope (TEM) that allows it to briefly (using a pulsed-laser-driven photocathode) operate at currents in excess of $10 \mathrm{~mA}$ while keeping the effects of condenser lens aberrations to a minimum. This modification allows real-space imaging of material microstructure with a resolution of order $10 \mathrm{~nm}$ over regions several $\mu \mathrm{m}$ across with an exposure time of $15 \mathrm{~ns}$. This is more than 6 orders of magnitude faster than typical video-rate TEM imaging. The key is the addition of a weak magnetic lens to couple the large-diameter high-current beam exiting the accelerator into the acceptance aperture of a conventional TEM condenser lens system. We show that the performance of the system is essentially consistent with models derived from ray tracing and finite element simulations. The instrument can also be operated as a conventional TEM by using the electron gun in a thermionic mode. The modification enables very high electron current densities in $\mu \mathrm{m}$-sized areas and could also be used in a non-pulsed system for high-throughput imaging and analytical TEM.
\end{abstract}

\section{Introduction}


LLNL-JRNL-422308

Now that modern aberration-corrected transmission electron microscopes (TEM) are routinely capable of spatial resolution on the sub-angstrom scale on static samples, the frontier of TEM instrument development is increasingly focused on high time resolution and in situ experimentation (the recent review articles by $\mathrm{Smith}^{1}$ and $\mathrm{Stach}^{2}$ contain extensive literature references). It is now possible to drive material processes with various combinations of heat, gaseous and fluid environments, and focused lasers and to capture the evolution using either conventional video-rate acquisition or much faster acquisition ranging from nanosecond-scale real-space imaging of unique irreversible events ${ }^{3-7}$ to femtosecond-scale diffraction, imaging, and spectroscopy of highly repeatable and/or reversible processes. ${ }^{8-10}$ The associated instrument development is at present very rapid, frequently bringing to light new challenges and solutions appropriate to the new physical regimes in which these instruments operate. Among those challenges is the operation of a factory-standard TEM column at the extremely high currents required for high-speed data acquisition.

The Dynamic Transmission Electron Microscope (DTEM) at Lawrence Livermore National Laboratory (LLNL) is a TEM modified for laser-driven in situ experimentation on the nanosecond scale (Fig. 1)..$^{3-7,11}$ A standard $200 \mathrm{keV}$ TEM (JEOL 2000FX) has been modified to allow a pair of nanosecond-scale pulsed lasers to enter the column. The first, called the sample drive laser, is focused to a spot on the sample with a variable diameter of order $100 \mu \mathrm{m}$. This laser initiates whatever process (e.g. rapid melting or other phase transformations, chemical reactions, laser ablation) is of interest to the experimenter. The second laser, called the cathode laser, is directed via a $45^{\circ}$ mirror onto a flat tantalum disc cathode situated at the site normally occupied by a standard thermionic cathode. This ultraviolet laser (211 nm wavelength) liberates a brief (10-15 ns) but very intense ( $\mathrm{mA}$, in comparison to the $\mu \mathrm{A}$ typical of a thermionic source) 
LLNL-JRNL-422308

pulse of photoelectrons. This pulse is accelerated to a potential of $200 \mathrm{kV}$, focused onto the electron-transparent sample, and imaged onto a camera just as in a standard TEM. This captures an image or diffraction pattern of the physical state of the sample at the time of arrival of the electron pulse. By varying the relative timing of the two lasers, one can thereby build up a history of the temporal evolution of the process of interest. The post-sample lenses behave nearly ${ }^{12,13}$ the same as they do in standard TEM operation (which we call continuous-wave $(\mathrm{CW})$ mode in contrast to the nanosecond-scale pulsed mode), enabling all of the standard TEM imaging and diffraction modes but with dramatically higher time resolution. The filament can also be heated, allowing the DTEM to operate in CW mode when needed.

The DTEM is capable of single-shot operation. That is, it is designed to contain enough electrons in each pulse to produce a complete image or diffraction pattern in one 10-15 ns exposure. This capability, necessary for the study of highly irreversible and unique events, distinguishes the DTEM from other approaches to ultrafast transmission electron microscopy (UFTEM) $)^{9,10}$ that gradually build up an image by accumulating electron counts over millions of cycles. The complementary multi-pulse approach is capable of extremely high time resolution when applied to physical processes that can be driven reversibly over a very large number of identical cycles. Unfortunately, many interesting processes are irreversible and/or will never temporally unfold the same way twice and therefore require single-shot capability for their study. Many phase transformations, chemical reactions, biological processes, and material processes (including defect motion and microstructural evolution) are of this type.

Previous publications $\mathrm{s}^{3-7,11-13}$ have described the basic principles and operation of the DTEM but have never fully documented the modifications to the electron optics and how these modifications have improved the imaging performance. In the present work, we report an 
LLNL-JRNL-422308

upgrade to the LLNL DTEM that enables a factor-of-20 (or more) increase in the current that can be delivered to the sample, with optimized lens coupling that reduces the effect of condenser lens aberrations to levels small compared to thermal emittance. This upgrade has dramatically increased the amount of information that can be obtained in a single 10-15 ns exposure of a rapidly evolving material process. We now have sufficient beam current and coherence to see features such as dislocations and stacking faults, with roughly $10 \mathrm{~nm}$ resolution, obtained over a region $5 \mu \mathrm{m}$ or more in diameter in a single nanosecond-scale snapshot. The availability of highquality single-shot images on this time and length enables in situ measurements of very fast, fundamental, irreversible material processes in a manner currently impossible on any other instrument.

\section{Technical Challenges}

Single-shot operation entails unique challenges that derive primarily from the finite brightness of the electron source. A high-quality image requires $\sim 10^{8}$ electrons to reach the camera, ${ }^{11}$ so that for a $10 \mathrm{~ns}$ exposure a current of at least $1.6 \mathrm{~mA}$ must reach the sample (and very likely more, due to post-sample apertures). This exceeds by orders of magnitude the nanoampere-scale currents used in conventional TEM. But the source only produces a finite current density and a finite normalized brightness (current per area per solid angle, corrected for

accelerating voltage $\left(\right.$ Reiser $^{14}$, chapter 3$)$ ), which is a constant in an ideal electron-optical system and thus cannot be improved regardless of how the beam is focused by the lens system. This means that, in order to collect such large currents, single-shot DTEM must typically use a larger source size and larger apertures than are used in conventional TEM. Improvements to the gun brightness can also help with this issue, but this topic is beyond the scope of the present article. 
In practice, lens aberrations can cause the effective brightness to degrade as the beam propagates from the source to the sample, a phenomenon called emittance growth (Reiser, ${ }^{14}$ chapter 6). The effects are particularly strong when large apertures are used in combination with large focusing field gradients such as those that occur in strong electric or magnetic lenses. The solution used in conventional TEM is shown in Fig. 2(a) (drawn for a thermionic source; a field emission system would look different in detail but similar in essence). A fixed aperture blocks most of the electrons before they enter the first condenser lens (C1). A second, variable aperture blocks the electrons that suffer strong condenser lens aberrations. By virtue of its relatively strong lenses, a conventional condenser system is capable of operating over a very wide range of magnification values for different applications. However these same strong lenses have large field gradients and will introduce excessive aberrations unless small apertures are used. Often well over $99 \%$ of the electrons generated at the source are deliberately blocked in order to maximize coherence and minimize the effects of aberrations. Since a thermionic source emits more current than a TEM operator will typically want to use in a conventional application, and since the improved coherence enables real-space imaging at atomic resolution, this design tradeoff is appropriate for conventional TEM. For a field emission source, the brightness is much higher but the current and source size are much smaller, and an electrostatic gun lens helps to control the beam collimation within the accelerator. Less of the current is blocked at fixed apertures, but the operation of the condenser lenses and the variable $\mathrm{C} 2$ aperture (the aperture near the center of the second condenser lens) is still much the same as it is in a thermioniccathode TEM.

DTEM cannot afford to operate with an electron optical design in which the vast majority of electrons are inevitably blocked if it is to achieve single-shot imaging. When it was first 
LLNL-JRNL-422308

deployed, the LLNL DTEM operated with a conventional C1/C2 condenser lens system, with the $45^{\circ}$ laser mirror inserted between the accelerator and the $\mathrm{C} 1$ lens, as shown in Fig. 2(b). The maximum current was obtained by widening all of the apertures in the system, while aberrations were reduced by reducing the strengths of the lenses (especially the $\mathrm{C} 1$ lens). This enabled single-shot imaging and diffraction experiments ${ }^{3,4}$ with as many as $10^{8}$ electrons per $10-15 \mathrm{~ns}$ pulse and spatial resolution better than $10 \mathrm{~nm}$. However, the instrument performance was still limited by a combination of spatial incoherence (arising primarily from gun and lens aberrations and the necessarily large source up to $\sim 0.5 \mathrm{~mm}$ in diameter) and poor signal-to-noise ratio (arising from the fact that, even with the widened apertures, over $90 \%$ of the electrons were still blocked by the $1 \mathrm{~mm}$ laser mirror aperture).

\section{Technical Solution: New Condenser Lens Design}

The solution is shown in Fig. 2(c). An extra condenser lens, dubbed C0 because it precedes the factory-standard condenser lenses $\mathrm{C} 1$ and $\mathrm{C} 2$, is inserted immediately below the accelerator. This lens was created from the condenser lens assembly from a JEOL 2000EX microscope nearly identical to the JEOL 2000FX on which the DTEM is based (Fig. 1). A long $(\sim 20 \mathrm{~cm})$ drift space is added below this new lens, giving the beam time to smoothly re-converge before reaching the laser mirror with its $1 \mathrm{~mm}$ aperture. The $\mathrm{C} 1$ lens coil and yoke from the JEOL 2000EX became the C0 lens. The polepiece was removed, simulations having shown that this would give the best performance in terms of lens aberrations. A two-dimensional magnetic deflector roughly halfway through this drift space facilitates alignment between $\mathrm{C} 0$ and $\mathrm{C} 1$. This deflector was integral to the parts obtained from the 2000EX and thus did not need to be separately fabricated. Implementation of the drift section required fabrication of a 10-cm-thick 
brass spacer with a hole drilled through the center to allow the electron beam to pass. This spacer was placed on top of the pre-existing (from the DTEM's initial deployment) custom-built brass section containing the $45^{\circ}$ mirror and vacuum ports for diagnostics.

The design was performed with a combination of analytical theory, numerical computation, and measurements of the instrument performance. The measurements benefited from a "knife-edge" diagnostic consisting of a flat metal edge just above the $45^{\circ}$ mirror that could be gradually inserted into the beam path using a high-precision step motor (Fig. 1). The beam current intercepted by the knife-edge was measured with a fast digital oscilloscope. This measurement provided both the temporal profile and the lateral spatial profile of the electron beam at that point in the column. It also allowed us to measure the spatial magnification and rotation between the knife-edge plane and either the $\mathrm{C} 2$ aperture plane or the sample plane, for any set of lens excitations. This was measured, for example, by recording the amount of knifeedge travel required to block the electrons at the extreme left and right edges of a $\mathrm{C} 2$ aperture of known size. Fitting the resulting data to a standard magnetic thin-lens model ${ }^{14}$ yielded a firstorder model of the DTEM's condenser lens system as well as an estimate of the virtual cathode position and diameter for various gun settings.

Third-order (aberration) terms were estimated by performing PARMELA ${ }^{15}$ and TRAK ${ }^{16}$ simulations of realistic magnetic lenses and accelerators similar to the actual hardware in the DTEM. These aberration terms can be presumed to have the correct scaling behavior but will differ from the exact machine performance by factors of order unity. Further, our knowledge of the column's electron optical characteristics was limited; focal lengths, lens positions, and fixed aperture positions were roughly estimated in some cases, and thick-lens effects and aberrations apart from spherical aberration were not modeled at all. The lens settings required for a paraxial 
crossover at the sample are very sensitive to some of these estimated parameters. The excitation of one of the lenses (specifically the condenser mini-lens above the objective lens) was used as a free parameter in the simulations to compensate for this. Since the simulations were merely intended to ensure that the aberration effects were negligible in comparison to the source's thermal emittance, and that the $\mathrm{C} 0$ lens could produce high throughput and an acceptable phase space profile at the sample, the model was satisfactory for our purposes.

The approximate third-order transfer function for each lens (as a function of its excitation setting) was modeled in a custom-written MATLAB ${ }^{17}$ simulation of the entire condenser lens system, with the electron beam's initial conditions made consistent with the results of the knifeedge measurements as well as the PARMELA simulations. The spherical aberration coefficient of each lens was set equal to the focal length, in the spirit of the degree of approximation discussed above. This model was used for the iterative process leading to the final $\mathrm{C} 0$ lens design. Detailed TRAK simulations of the exact $\mathrm{C} 0$ lens geometry were performed to ensure that the actual lens would function as required.

The $\mathrm{C} 0$ lens has an extremely wide bore $(\sim 5 \mathrm{~cm})$ and long focal length $(\sim 15 \mathrm{~cm})$, more than a factor of ten larger than the same quantities for a typical TEM condenser lens. This is an appropriate design to minimize the effect of aberrations when a lens is intended to smoothly reconverge a very broad, moderately divergent beam. This is a very different role from those of the objective and projector lenses, which usually provide the best performance with small polepiece gaps and short focal lengths. The magnetic field and magnetic field gradients in the C0 lens are quite small, and it can capture and re-focus even a very wide beam (up to $5 \mathrm{~mm}$ diameter) without introducing large aberration effects. The TRAK and MATLAB simulations indicate that the aberration-induced emittance growth created by $\mathrm{C} 0$ is negligible compared to 
LLNL-JRNL-422308

the thermal emittance generated at the cathode. In other words, the lens is able to transfer essentially the full brightness of the source to the entrance of the $\mathrm{C} 1$ lens; it is a nearly ideal coupling lens. The beam is less than $1 \mathrm{~mm}$ in diameter as it enters $\mathrm{C} 1$, so if the system is well aligned then $\mathrm{Cl}$ 's aberrations will also introduce relatively little emittance growth.

These predictions are borne out in practice. Fig. 3a shows a typical illumination spot for the DTEM operated in conventional mode (i.e. with thermionic emission and the C0 lens off) without a $\mathrm{C} 2$ aperture, focused to a paraxial crossover. The spot consists of a very intense central region surrounded by a much broader halo of aberrated electrons. The halo is asymmetric due to difficult-to-correct mechanical misalignment of fixed apertures. Most of the aberrations that yield the halo are produced by the $\mathrm{C} 1$ lens (as was verified in the simulations and by noting the effect of the $\mathrm{C} 1$ excitation on the measured halo), and the aberrated and asymmetrically apertured electrons can be removed by inserting a C2 aperture (Fig. 3c). This situation is familiar to all TEM operators. The transverse phase-space plot in Fig. 3e clarifies the situation: Lens aberrations cause a marked sigmoidal curvature in the calculated phase space distribution, making it impossible to simultaneously focus the paraxial and marginal electrons. The $\mathrm{C} 2$ aperture eliminates all but the nearly-paraxial electrons, enabling a tight focus (limited by the beam temperature and source size and not by aberrations) while also providing a direct way to control the angular range (which is important for producing high-resolution images and diffraction patterns).

Figs. 3(b,d,f) show an analogous situation using the $\mathrm{C} 0$ lens to collect a much larger fraction of the current. This time the sigmoidal curvature is negligible in comparison with the effects of the finite beam temperature. The $\mathrm{C} 2$ aperture eliminates a small amount of beam asymmetry (probably arising from minor misalignments) and again allows control of the 
convergence angle, but its previous role in eliminating aberrated electrons is no longer its primary purpose. The throughput (fraction of collected electrons) is also larger by a factor of order 20-100 (depending on lens and aperture settings as well as gun alignment). Since brightness is conserved, the phase space area (which is proportional to the emittance) in Fig. $3 \mathrm{f}$ is also larger by a factor of order $20^{1 / 2}$ to $100^{1 / 2}$. This is inevitable (given conservation of brightness) and is consistent with the purpose of the $\mathrm{C} 0$ lens, namely to greatly increase the current available for $\mu \mathrm{m}$-scale illuminated areas without introducing excessive aberrations. We have achieved total current throughputs approaching 100\% (within experimental uncertainty) in the DTEM by adjusting $\mathrm{C} 0, \mathrm{C} 1$, and $\mathrm{C} 2$ and eliminating as many apertures as possible. The results in Fig. 3(b,d) use $\mathrm{C} 0$ in a slightly defocused condition, reducing throughput to just under $50 \%$ (with no $\mathrm{C} 2$ aperture) while making the instrument easier to align and eliminating many of the electrons that are badly aberrated by the electric field gradients near the gun itself. Owing to space charge effects, these gradients are different in pulsed and CW modes. Throughput is measured by comparing the emission current (i.e. total high voltage current minus the dark and biasing current measured with the filament heat off) to the current measured with a Faraday cup placed in the TEM camera chamber (Fig. 1). The calculated spatial profiles in Fig. $3 \mathrm{~g}$ seem to match the experimental measurements quite well, apart from a factor of $\sim 3$ in the intensity of the low-current ( $\mathrm{C} 0$ off) no-aperture case. This discrepancy may be partly explained by the misalignment apparent in Fig. 3a (throughput being very sensitive to gun alignment when the instrument is operated in this way), but is also probably partly derived from the known limitations and deliberate simplifications in the model as already discussed; the model really is intended to capture the lens transfer properties in essence and not in detail. In any case, Fig. $3 g$ 
LLNL-JRNL-422308

indicates that the operation of the $\mathrm{C} 0$ lens in the context of the complete condenser lens system is fairly well understood.

An example of the detailed ray-tracing simulation for operating parameters fairly typical of DTEM 15 ns single-shot operation (and roughly similar, though surely not identical in detail, to the measurement in Fig. 3(d)) is shown in Fig. 4 and Table I. As with any TEM, the user can alter the lens focal lengths and C2 aperture size at will over very wide ranges, so the calculation presented here is merely meant as an example. Note the rms geometrical emittance values before and after each lens. The emittance growth introduced by $\mathrm{C} 0$ is negligible. The worst offender in the system is the second gap of the $\mathrm{C} 1$ lens, the aberrations of which increase the geometrical emittance by nearly $1 \%$ (from 3.820 to $3.853 \mathrm{~nm}$-rad; note the increase in sigmoidal curvature between crossovers $\mathrm{B}$ and $\mathrm{C})$. The $\mathrm{C} 2$ aperture very slightly ( $\sim 2 \%$ change) improves the rms brightness (which scales as current per mean squared emittance) by eliminating the electrons most badly aberrated by the second gap of $\mathrm{C} 1$. After this, the transverse phase space area is essentially fixed, and C2, CM, and the objective lens prefield merely determine the shape of the phase space when it hits the sample.

A discussion of the scaling of spherical aberration will help to demonstrate why the $\mathrm{C} 0$ lens is so effective. Spherical aberration prevents a point object from focusing to a single image point. Instead the point will produce a disk of least confusion of radius $r=C_{S} \alpha^{3} / 4$, where $C_{S}$ is the spherical aberration coefficient of the lens (similar in magnitude to the focal length $f$ ) and $\alpha$ is the convergence angle (Reiser, ${ }^{14}$ p. 108) (note that various authors' definitions will differ by factors of order unity; also note that, for the moment, we are only considering the $\mathrm{C} 0$ lens and not its role in the complex lens system). Since the $\mathrm{C} 0$ lens is not operating near the diffraction limit (the crossover is many $\mu \mathrm{m}$ in diameter, while the electron wavelength is $2.5 \mathrm{pm}$ and $\alpha \sim$ 
LLNL-JRNL-422308

0.005 radian), the aberration-limited crossover size is obtained by making $\alpha$ as small as possible. This means making the drift section as long as possible. Although this means the lens must be made weaker and $C_{S}$ will thereby increase, the cubic dependence of $r$ on $\alpha$ more than compensates, and $r$ decreases rapidly as the drift section length is increased. Eventually diminishing returns are reached, as the $\mathrm{C} 0$ aberrations cease to be the dominant factor limiting the performance, as is apparent in Fig. 3f. The aberrations cause the sigmoidal curvature of the dark line and their effects are quite small in comparison to the effects of finite beam temperature as indicated by the spread of the scatter plot. In other words, while the aberrations of the $\mathrm{C} 0$ lens do introduce a slight blur in the following crossover, this blur is negligible compared to the diameter of the crossover caused by the finite source size and beam temperature. The emittance growth introduced by $\mathrm{C} 0$ is negligible compared to the thermal emittance.

In light of these diminishing returns, we found that a $20 \mathrm{~cm}$ drift section after $\mathrm{C} 0$ (which increased the total column height by nearly $30 \mathrm{~cm}$ ) was an ideal compromise between the electron optical performance and practical considerations in the implementation. These considerations included seismic stability, the difficulty of extending the vacuum system and hydraulic gun lift, and ultimately the height of the room containing the instrument. There are also questions of the effect of space charge on the beam propagation, which may be significant for the DTEM's multi-milliampere pulses. However, simulations (using PARMELA and semianalytical models $^{18,19}$ ) showed that the relevant effects are quite small for the 10-15 nanosecond pulses used in DTEM. At most, the space charge effects on the condenser lens behavior simply force the user to slightly refocus the lenses between pulsed and CW modes (which is required anyway, since the virtual source position changes as the gun is operated in a different mode), while the associated space-charge-induced aberrations and broadening of pulse durations and 
LLNL-JRNL-422308

energy spreads are usually negligible. These simulation predictions are consistent with our

experience in day-to-day operation of the microscope. Stochastic blurring effects ${ }^{12}$ are of course still present; the $\mathrm{C} 0$ lens does not correct this.

\section{Results}

We have already shown that, in CW mode, the new lens system allows for efficient, lowaberration transfer to the sample plane of most of the brightness and current produced by the electron gun. This is accomplished with a simple modification, namely the addition of a new weak lens, and does not require aberration correction. Since the C0 lens excitation is continuously variable, the experimenter can obtain at will any desired level of performance ranging from that of a standard thermionic TEM (with the $\mathrm{C} 0$ lens shut off or turned very low) to the extreme case in which nearly every electron leaving the gun reaches a $\mu \mathrm{m}$-scale area on the sample. This gives the experimenter enormous flexibility to trade off signal levels against coherence for any given experiment, without having to worry about the effects of condenser lens aberrations. In practice, we find that it is often better to fix the $\mathrm{C} 0$ excitation setting and instead vary the $\mathrm{C} 1$ excitation and $\mathrm{C} 2$ variable aperture size. The electron optical results are similar, but the alignment procedures are simplified, and holding the $\mathrm{C} 0$ strength constant also allows us to ensure that the $\mathrm{x}$-ray generation at the $45^{\circ}$ mirror plane is kept to a minimal level. X-ray generation can be significant, since the column is operating at a current much higher than anticipated in its original design. Any implementation of a $\mathrm{C} 0$-like lens must include proper attention to this fact, including full x-ray surveys throughout the range of operating parameters.

The added lens also greatly improved the performance and flexibility in the DTEM's unique single-shot pulsed mode. Before installing the $\mathrm{C} 0$ lens, the LLNL DTEM was incapable 
LLNL-JRNL-422308

of putting more than $10^{8}$ electrons into a $15 \mathrm{~ns}$ pulse, and even this could only be accomplished by accepting large reductions in coherence due to gun and lens aberrations. Now, the DTEM can routinely put $2 \times 10^{9}$ electrons into each 15 ns pulse (as measured by a Faraday cup just above the projector lens), an increase by a factor of 20 , without significantly increasing condenser lens aberration effects. It appears that, just as in CW mode, most of the emittance comes from the inevitable thermal emittance and electrostatic lens aberrations at the source. The spatial resolution in an image has hardly changed (being of order $10 \mathrm{~nm}$ ), since spatial resolution under low signal-to-noise ratio conditions is determined by an optimized interplay of beam brightness and objective lens aberrations, ${ }^{11}$ and neither of these parameters has changed. However, our optimum spatial resolution can now be achieved over a much wider area; the amount of information per image has greatly improved. Previously, the DTEM's best short-pulse resolution could only be obtained on sub- $\mu \mathrm{m}$ areas, ${ }^{4}$ but now it can be achieved on regions $5 \mu \mathrm{m}$ or even 10 $\mu \mathrm{m}$ in diameter, depending on the contrast of the sample.

Fig. 5 is an example, showing short-pulse and CW images of a region of stainless steel that includes stacking faults and dislocations. The $15 \mathrm{~ns}$ pulsed exposure contains much of the microstructural information that is evident in the long-exposure $\mathrm{CW}$ image. The signal-to-noise ratio is of course not as good, but this is to be expected given the enormous difference in exposure times. Typically the operator would use the long-exposure image to help in unambiguous interpretation of the information in the short-pulse image; this is a major advantage of having retained the conventional $\mathrm{CW}$ operating mode as an option in the DTEM. The images in Fig. 5 are of a static sample and are meant as proof of a principle: Direct, single-shot observation of microstructural features is now possible, on a scale of nanometers and nanoseconds, over areas large enough to be relevant for the understanding of material processes. 
LLNL-JRNL-422308

Other results ${ }^{5}$ using the DTEM with the C0 lens have shown that the instrument is capable of capturing images of fast-evolving, transient, nanometer-scale structures that cannot be seen in conventional TEM.

\section{Conclusion}

With the development of the C0 coupling lens, the performance of the DTEM is no longer limited by condenser lens aberrations and the unavoidable loss of electrons at fixed apertures. The majority of the current generated by the electron gun can be faithfully transferred to the sample without significant degradation of effective brightness. The upgrade was relatively simple, since most of the electron optical column was unmodified and could still be operated in the usual way. The exception is in the gun alignment (which now must be performed iteratively and with somewhat nonstandard procedures) and the $\mathrm{C} 1$ lens excitation, which is often turned down to a level much lower than its standard operating range, since it normally handles a diverging beam rather different from the converging beam produced by $\mathrm{C} 0$ (Figs. 2, 4). After $\mathrm{C} 1$, the beam paths are practically the same as they are in the factory-standard operating modes. In addition, the performance and behavior of the unmodified instrument can be recovered to a good approximation simply by adjusting the excitations of the condenser lenses and performing minor realignments.

While we developed this modification for the purposes of nanosecond-scale single-shot imaging, it has not escaped our attention that this same modification could be valuable for conventional TEMs. In conventional thermionic mode, the newly-modified DTEM can put over $4 \mu \mathrm{A}$ of current into a fairly uniform spot less than $1 \mu \mathrm{m}$ in diameter at the sample plane (as measured by the Faraday cup in Fig. 1). This is an enormous beam current by TEM standards. Provided the sample can survive such heavy irradiation, such a system should enable extremely 
short acquisition times for energy-dispersive $\mathrm{X}$-ray and electron energy-loss spectroscopies as well as conventional and energy-filtered imaging. Not all applications require the extremely fine probes and high beam coherence of modern TEM's. For such applications, a lens similar to our C0 lens could dramatically reduce acquisition times and increase data throughput.

\section{Acknowledgments}

This work performed under the auspices of the U.S. Department of Energy, Office of Basic Energy Sciences, Division of Materials Science and Engineering, by Lawrence Livermore National Laboratory under Contract DE-AC52-07NA27344.

\section{References}

${ }^{1}$ D. J. Smith, Materials Today, Microscopy Special Issue, 30 (January 21, 2009)

${ }^{2}$ E. A. Stach, Materials Today, Microscopy Special Issue, 50 (January 21, 2009)

${ }^{3}$ J. S. Kim, T. LaGrange, B. W. Reed, M. L. Taheri, M. R. Armstrong, W. E. King, N. D.

Browning, and G. H. Campbell, Science 321, 1472 (2008)

${ }^{4}$ T. LaGrange, G. H. Campbell, B. W. Reed, M. L. Taheri, J. B. Pesavento, J. S. Kim, and N. D. Browning, Ultramicroscopy 108, 1441 (2008)

${ }^{5}$ T. LaGrange, D. S. Grummon, B. W. Reed, N. D. Browning, W. E. King, and G. H. Campbell, Appl. Phys. Lett. 94, 184101 (2009)

${ }^{6}$ T. LaGrange, G. H. Campbell, P. E. A. Turchi, and W. E. King, Acta Mat. 55, 5211 (2007)

${ }^{7}$ T. LaGrange, M. R. Armstrong, K. Boyden, C. G. Brown, G. H. Campbell, J. D. Colvin, W. J. DeHope, A. M. Frank, D. J. Gibson, F. V. Hartemann, J. S. Kim, W. E. King, B. J. Pyke, 
B. W. Reed, M. D. Shirk, R. M. Shuttlesworth, B. C. Stuart, B. R. Torralva, and N. D. Browning, Appl. Phys. Lett. 89, 044105 (2006)

${ }^{8}$ B. J. Siwick, J. R. Dwyer, R. E. Jordan, and R. J. D. Miller, Science 302, 1382 (2003)

${ }^{9}$ V. A. Lobastov, R. Srinivasan, and A. H. Zewail, Proc. Natl. Acad. Sci. 102, 7069 (2005)

${ }^{10}$ F. Carbone, O.-H. Kwon, and A. H. Zewail, Science 325, 181 (2009)

${ }^{11}$ B. W. Reed, M. R. Armstrong, N. D. Browning, G. H. Campbell, J. E. Evans, T.

LaGrange, and D. J. Masiel, Microscopy and Microanalysis 15, 272 (2009)

${ }^{12}$ M. R. Armstrong, B. W. Reed, B. R. Torralva, and N. D. Browning, Appl. Phys. Lett. 90, $114101(2007)$

${ }^{13}$ M. R. Armstrong, K. Boyden, N. D. Browning, G. H. Campbell, J. D. Colvin, W. J.

DeHope, A. M. Frank, D. J. Gibson, F. Hartemann, J. S. Kim, W. E. King, T. B. LaGrange, B. J. Pyke, B. W. Reed, R. M. Shuttlesworth, B. C. Stuart, and B. R. Torralva Ultramicroscopy 107, $356(2007)$

${ }^{14} \mathrm{M}$. Reiser, Theory and Design of Charged Particle Beams. New York: John Wiley \& Sons (1994)

${ }^{15}$ L. M. Young, LANL Report No. LA-UR-96-1835

${ }^{16}$ Field Precision LLC, http://www.fieldp.com

${ }^{17}$ The Mathworks, http://www.mathworks.com

${ }^{18}$ B. W. Reed, J. Appl. Phys. 100, 034916 (2006)

${ }^{19}$ P. Kruit and G. H. Jansen, Space Charge and Statistical Coulomb Effects. In Handbook of Charged Particle Optics, Orloff, J. (Ed.), pp. 275-318. Boca Raton: CRC Press (1997) 
LLNL-JRNL-422308

\section{Figure Captions}

Fig. 1 (color online). Photograph and same-scale schematic of the LLNL Dynamic Transmission Electron Microscope. Two pulsed lasers are interfaced with a conventional TEM to produce a unique in situ TEM capable of capturing a complete image or diffraction pattern in a single 10$15 \mathrm{~ns}$ exposure. To the factory-standard JEOL 2000FX TEM we have added the C0 lens section (salvaged from a similar instrument and modified), two brass sections (including a spacer section and a section that includes a laser window, a 45 degree laser mirror, and a motorized knife edge), a path for a laser to directly drive the sample, and a Faraday cup in the camera chamber to measure beam current. The instrument can also operate as a conventional thermionic-cathode TEM.

Fig. 2. Ray paths for the electron guns and condenser lenses used in conventional and dynamic TEM. The paths are simplified; the real DTEM has a double-gap C1 lens, a condenser mini-lens, and a strong objective lens pre-field not represented in the figure. (a) Conventional ("CW") TEM with a thermionic cathode. The source produces more current than needed, and aberrated electrons are blocked at apertures. (b) DTEM before the $\mathrm{C} 0$ upgrade. A laser mirror with an aperture drilled through the center is inserted below the accelerator. Because DTEM requires high currents, the condenser lenses are operated in a mode that retains many aberrated electrons. (c) DTEM after the upgrade. The new C0 lens changes the coupling so that nearly all of the electrons pass through the mirror aperture, while emittance growth from condenser lens aberrations is negligible compared to the gun's thermal emittance. The user can now use as much or as little of the beam as desired, as dictated by experimental needs and the brightness of the source. 
Fig. 3. Illustrating the effects of the $\mathrm{C} 0$ lens and $\mathrm{C} 2$ variable aperture on the spatial profile of a beam focused close to a paraxial crossover at the sample plane. (a-d) Measured images divided by the gun emission current to give a value proportional to the throughput (displayed on base-10 logarithmic intensity scales). (e-f) Simulations of the transverse phase space profiles including representative scatter plots at finite beam temperature and black lines at zero temperature. (a,c,e) $\mathrm{C} 0$ lens off (conventional operation). (b,d,f) C0 lens on (high-current operation). (a,b,open circles and dashed lines in (e,f)) no C2 aperture. (c, filled circles and solid line in (e)) $100 \mu \mathrm{m}$ C2 aperture. (d, filled circles and solid line in (f)) $200 \mu \mathrm{m} \mathrm{C} 2$ aperture. (g) Transverse beam profiles (scaled projections onto the $\mathrm{x}$ axis of data underlying (a-f)). Peaks of measured and calculated $\mathrm{C} 0$ on/no aperture curves were matched to each other; otherwise there were no free parameters in the intensity scaling.

Fig. 4: An example ray trace for typical lens and aperture settings used in 15 ns single-shotimaging operation at $200 \mathrm{kV}$ in the DTEM. Drawing is to scale, with an aspect ratio distortion indicated by the scale bars. Non-normalized RMS emittance values $\varepsilon$ (a constant of the motion for free-space propagation, inversely related to the spatial coherence of the beam; Reiser, ${ }^{14}$ p. 57) are indicated after each lens. Inset graphs show phase-space plots (using the same conventions as in Fig. 3) for (A) the entrance to $\mathrm{C} 0$, (B) the first crossover, and (C) the second crossover, while the profile at the sample is similar to that shown in Fig. 3f. This is just an example; since lens strengths and aperture sizes can be easily varied, a very wide range of operating parameters is available. 
Fig. 5: Images of microstructure, including dislocations and stacking faults, in stainless steel. Much of the microstructure in the conventional exposure (right) is also visible in the pulsed exposure (left) despite the difference in exposure times of nearly 8 orders of magnitude. Before installation of the $\mathrm{C} 0$ lens it was impossible for the DTEM to acquire nearly so much information in a single $15 \mathrm{~ns}$ pulse. These images were not processed apart from standard dark count and flat-field corrections. 
Table I: First-order thin-lens transfer properties for the ray trace example shown in Fig. 4. Note the objective lens (OL) is a thick lens with the sample centered within it, so that the thin lens model is only a very rough approximation for this lens. However, small adjustments of the condenser mini-lens (CM) strength still allowed a calculation of realistic profiles at the sample plane (see Fig. 3). Positions and magnifications are measured from the virtual cathode crossover (VCC), which is taken as the object upon which the lens system acts. Image positions in parentheses are virtual, while the others are real crossover points in the column. The magnification of each lens is shown, along with the cumulative magnification from the VCC to the current image position.

\begin{tabular}{cccccc}
\hline \hline Element & Position & $f$ & Image Position & $m_{\text {lens }}$ & $m_{\text {cumulative }}$ \\
& $(\mathrm{mm})$ & $(\mathrm{mm})$ & $(\mathrm{mm})$ & & \\
\hline $\mathrm{VCC}$ & 0 & & 0 & & 1 \\
$\mathrm{C} 0$ & 515 & 162.9 & $(753)$ & -0.463 & -0.463 \\
$\mathrm{C} 1 \mathrm{~A}$ & 745 & 13.2 & 750 & 0.613 & -0.284 \\
$\mathrm{C} 1 \mathrm{~B}$ & 779 & 13.2 & 803 & -0.843 & 0.239 \\
$\mathrm{C} 2$ & 842 & 54.3 & $(708)$ & 3.46 & 0.828 \\
$\mathrm{CM}$ & 960 & 48.8 & $(1020.5)$ & -0.240 & -0.199 \\
$\mathrm{OL}$ & 1020 & 2.3 & 1020.4 & 0.827 & -0.164 \\
\hline \hline
\end{tabular}


Figure 1
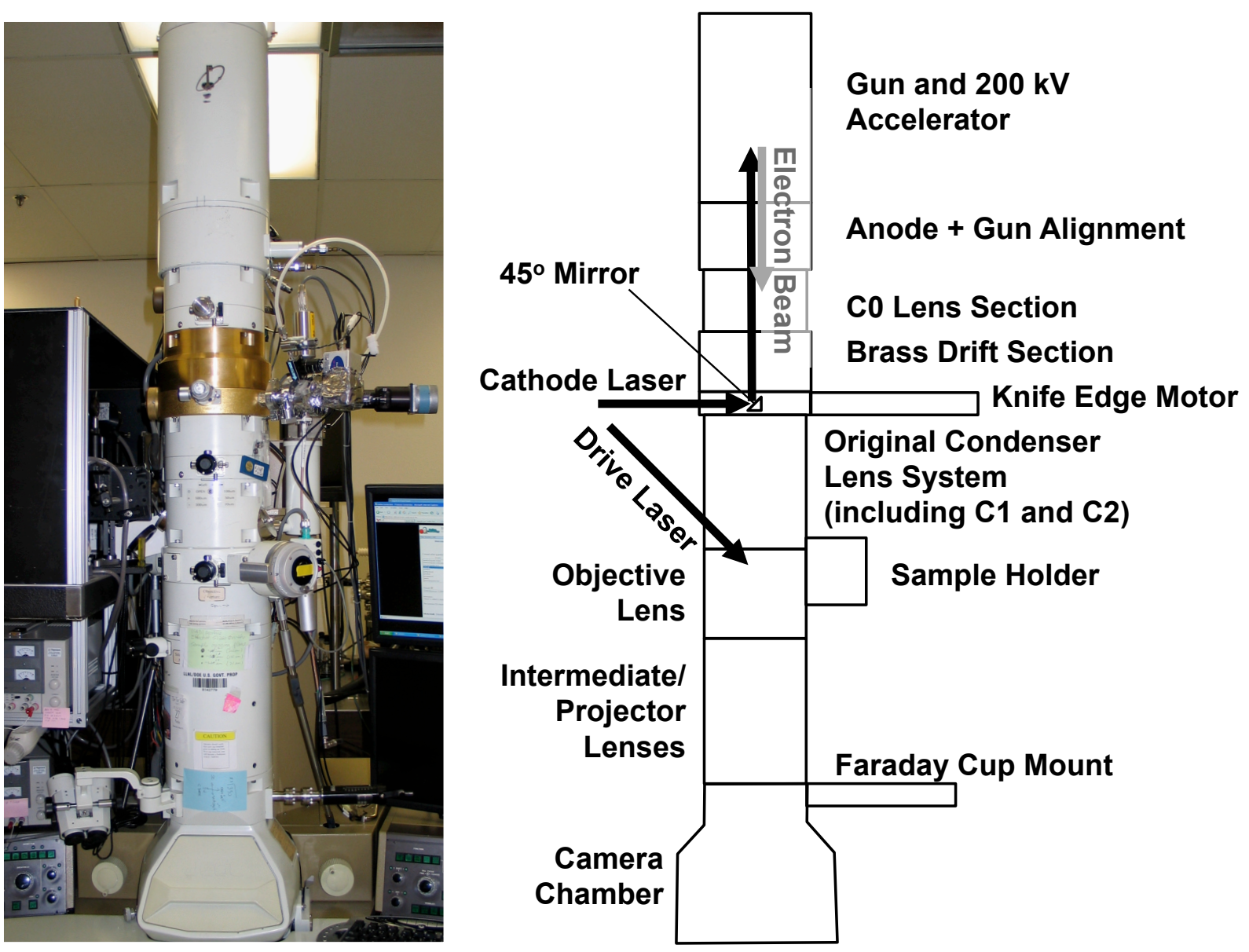
Figure 2
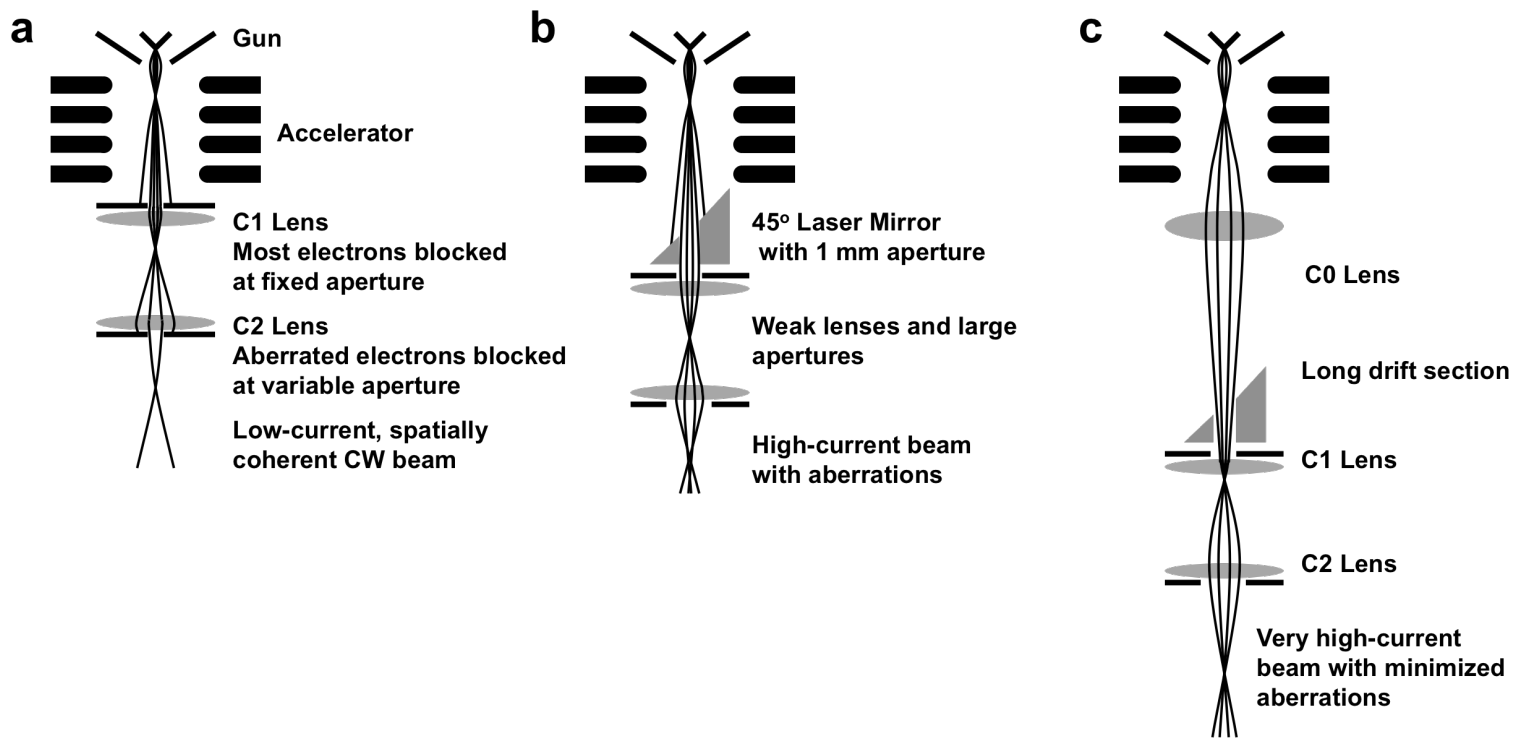
Figure 3
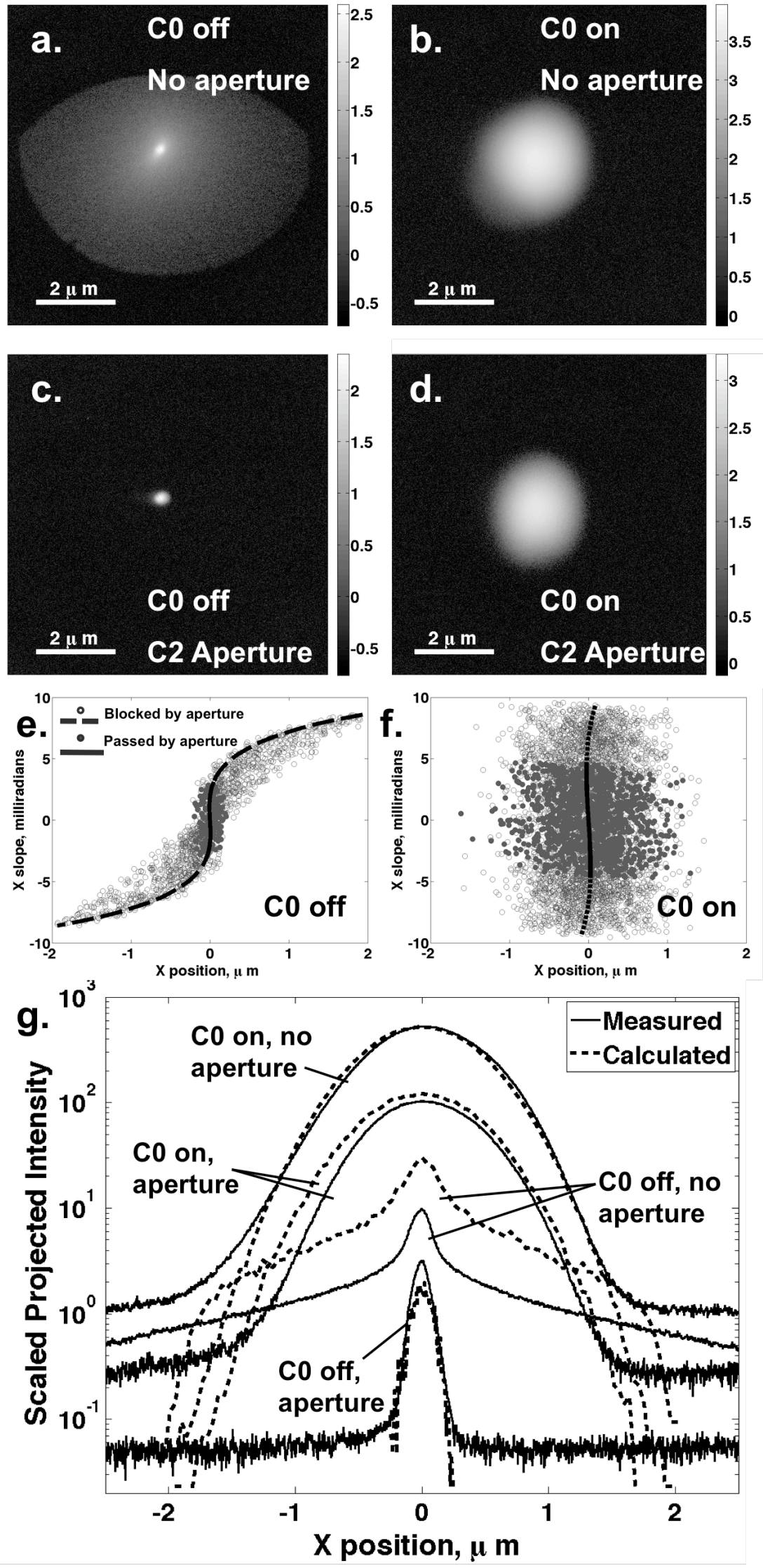
Figure 4

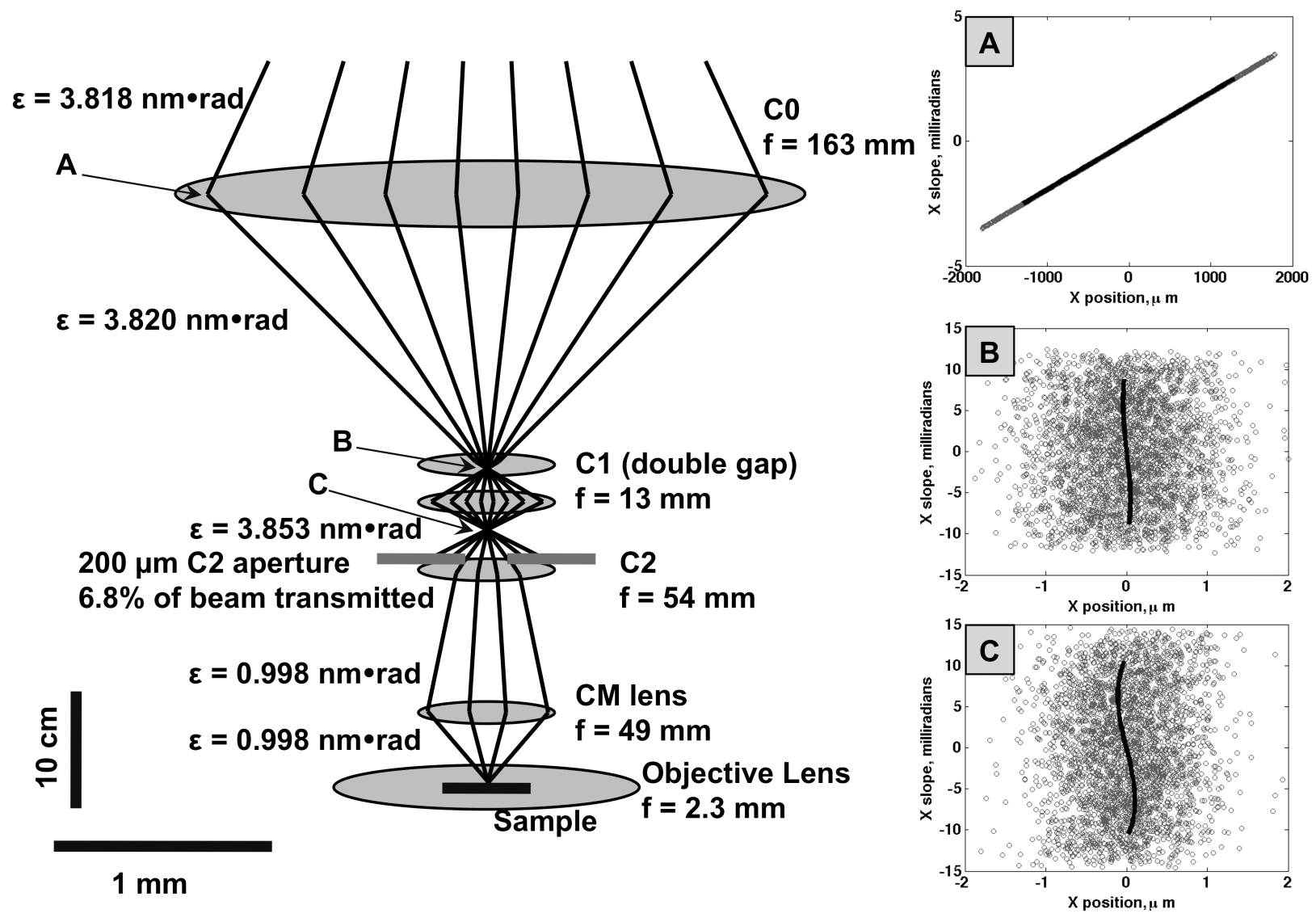


Figure 5
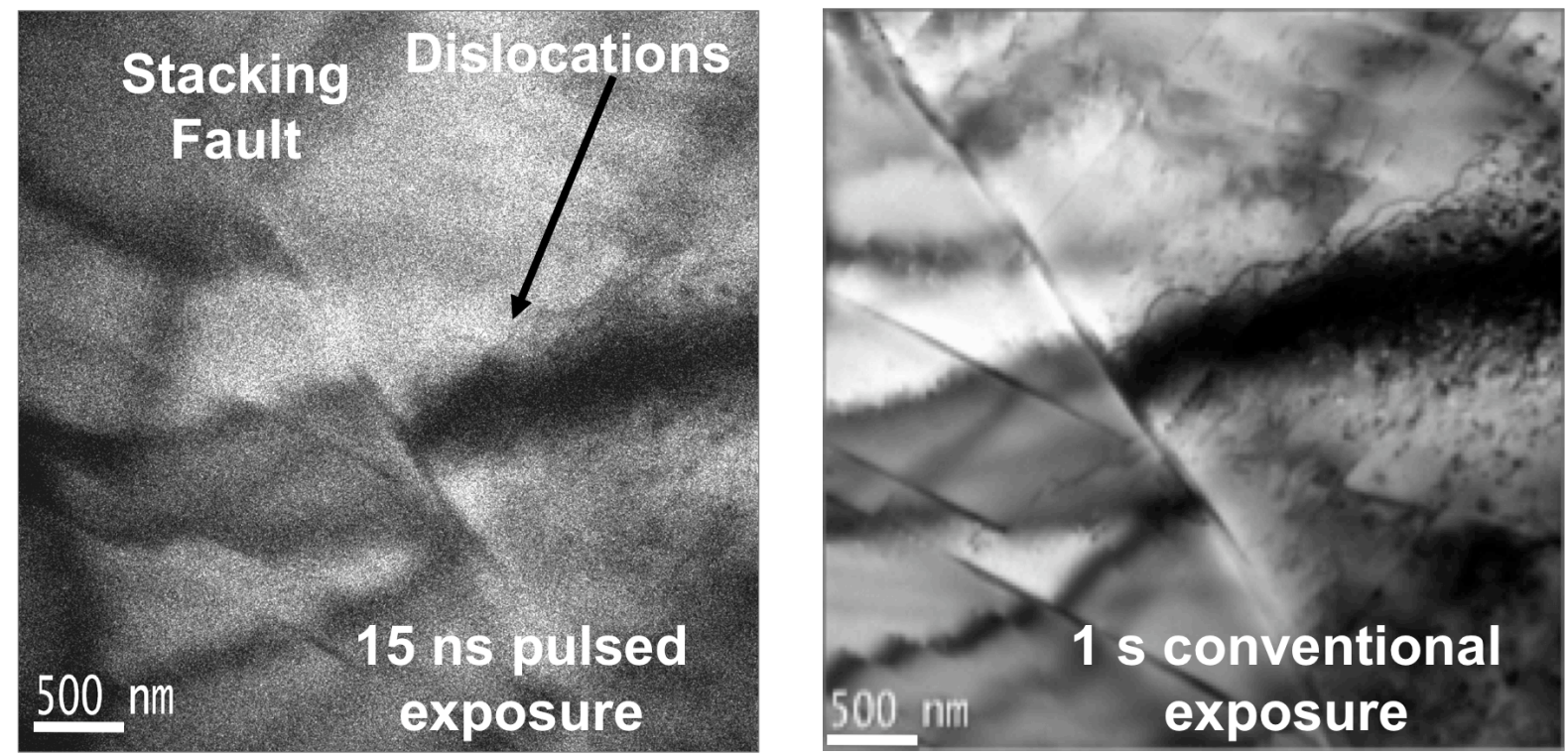